\title{
A tight upper bound of the lumped disk seek time for the Scan disk scheduling policy
}

\author{
Yen-Jen Oyang ${ }^{1}$ \\ Department of Computer Science and Information Engineering, National Taiwan University, Taipei, Taiwan
}

Communicated by K. Ikeda; received 29 September 1994

\begin{abstract}
A tight upper bound of the lumped disk seek time for the Scan disk scheduling policy is presented. The tight upper bound is based on an accurate disk seek time model. One of the most important applications of the tight upper bound is in multimedia storage system design. Without a tight upper bound based on an accurate disk seek time model, the designers of multimedia storage systems would be forced to use more conservative approaches, i.e. loose upper bounds, to guarantee satisfaction of real-time requirements. This, in turn, would result in underutilization of disk bandwidth.
\end{abstract}

Keywords: Information retrieval; Disk scheduling; Modeling; Real-time systems

\section{Introduction}

The Scan policy is a popular scheme for scheduling disk head movement $[1,5,2]$. The basic mechanism of the Scan policy is that the queued $\mathrm{I} / \mathrm{O}$ requests are scheduled according to the physical locations of the data so that these $I / O$ requests can all be serviced when the disk head sweeps unidirectionally (inward or outward) across the disk surface. Though the idea is quite simple, the Scan policy has been gaining more attention than before due to emerging of multimedia storage systems $[6,7,3]$.

In the design of multimedia storage systems, an essential issue is how to guarantee uninterrupted delivery of continuous data. This means that the systems must meet certain real-time requirements. Without a tight upper bound based on an accurate disk seek time model, the designers of multimedia storage systems

\footnotetext{
${ }^{1}$ Email: yjoyang@csie.ntu.edu.tw.
}

would be forced to use more conservative approaches, i.e. loose upper bounds, to meet the real-time requirements. This, in turn, would result in underutilization of disk bandwidth. For example, the system developed by researchers at University of California, San Diego [6] uses the maximum seek time, which is the time the disk head takes to sweep across the entire disk surface, as the upper bound of a single disk seek. The GSS scheme proposed by Chen, Yu, and Kandlur [7] uses a linear model for disk seek time. However, a linear model could mean a wide range off of the actual seek time. For example, the linear equation used by Ruemmler and Wilkes [4] for a long seek of the HP97560 hard disk predicts that

$$
8.00+0.008 * 25=8.2 \text { milliseconds }
$$

is needed to move the head 25 cylinders away. On the other hand, the accurate model presented in the same paper says only 5.24 milliseconds is needed.

This paper presents a tight upper bound of the 
lumped disk seek time for the Scan policy based on an accurate model [4]. In the model, a seek is composed of

- a speedup, where the head is accelerated until it reaches half of the seek distance or a fixed maximum velocity;

- a coast for long seeks, where the head moves at its maximum velocity;

- a slowdown, where the head is brought to rest close to the desired track;

- a settle, where the disk controller adjusts the head to access the desired location;

Short seeks spend almost all the time in the constantacceleration phases and, as a result, the seek time is proportional to the square root of the seek distance plus the settle time. Long seeks spend some of the time moving at a constant speed, taking time that is proportional to distance plus a constant overhead. Accordingly, the general form of the model is

seek time $= \begin{cases}c_{1}+c_{2} \sqrt{d} & \text { if } d \leqslant D, \\ c_{3}+c_{4} d & \text { if } d>D,\end{cases}$

where $D$ is a constant defining the boundary of the two formulas above and $d$ is the distance of the seek in numbers of cylinders.

The tight upper bound derived in this paper states that, when a disk head scans across a region, the lumped seek time is maximized when the stops are evenly apart. As the discussion above indicates, the tight upper bound can be applied to improve disk bandwidth utilization in the design of multimedia storage systems.

\section{Mathematical proof}

This section presents the mathematical proof of the tight upper bound. In the subsequent discussion, $\mathrm{N}^{+}$ denotes the set of all positive integers and $R$ denotes the set of all real numbers.

Lemma 1. Let $h: N^{+} \rightarrow R$ be a function with $h(i) \geqslant$ $h(j)$ for all $i, j \in N^{+}$and $i<j$. Then, for an $n_{0} \in N^{+}$ and two series of positive integers $l_{1}, l_{2}, \ldots, l_{p}$ and $m_{1}, m_{2}, \ldots, m_{q}$, we have

$$
\sum_{i=1}^{p} \sum_{k=1}^{l_{i}} h\left(n_{0}-k\right) \geqslant \sum_{j=1}^{q} \sum_{k=1}^{m_{j}} h\left(n_{0}+k-1\right),
$$

if

$\sum_{i=1}^{p} l_{i}=\sum_{j=1}^{q} m_{j} \quad$ and $\quad n_{0}>l_{i}$

for all $1 \leqslant i \leqslant p$.

Proof. Since $h(i) \geqslant h(j)$ for all $i, j \in N^{+}$and $i<j$, we have

$$
\begin{aligned}
& \sum_{i=1}^{p} \sum_{k=1}^{l_{i}} h\left(n_{0}-k\right) \\
& \geqslant \sum_{i=1}^{p} l_{i} h\left(n_{0}\right)=h\left(n_{0}\right) \sum_{i=1}^{p} l_{i} \\
& =h\left(n_{0}\right) \sum_{j=1}^{q} m_{j}=\sum_{j=1}^{q} m_{j} h\left(n_{0}\right) \\
& \geqslant \sum_{j=1}^{q} \sum_{k=1}^{m_{j}} h\left(n_{0}+k-1\right) .
\end{aligned}
$$

Lemma 2. Let $f: N^{+} \rightarrow R$ be a function and $f^{\prime}(n)=f(n+1)-f(n)$ for all $n \in N^{+}$. If $f^{\prime}(i) \geqslant$ $f^{\prime}(j)$ for all $i<j$, then for a positive integer $n_{0}$ and a series of integers $d_{1}, d_{2}, \ldots, d_{s}$ with $\sum_{i=1}^{s} d_{i}=0$ and $n_{0}+d_{i} \geqslant 1$ for all $d_{i}$, we have

$s f\left(n_{0}\right) \geqslant \sum_{i=1}^{s} f\left(n_{0}+d_{i}\right)$.

Proof. Without loss of generality, assume

$d_{1}, d_{2}, \ldots, d_{p}<0$ and $d_{p+1}, d_{p+2}, \ldots, d_{s} \geqslant 0$.

Let

$q=s-p$,

$l_{i}=-d_{i} \quad$ for $1 \leqslant i \leqslant p$,

$m_{j}=d_{p+j}$ for $1 \leqslant j \leqslant q$.

Then, we have

$\sum_{i=1}^{p} l_{i}=\sum_{j=1}^{q} m_{j}$ 
and

$$
\begin{aligned}
\sum_{i=1}^{s}\left[f\left(n_{0}+d_{i}\right)-f\left(n_{0}\right)\right] & \\
= & \sum_{i=1}^{p}\left[f\left(n_{0}-l_{i}\right)-f\left(n_{0}\right)\right] \\
& +\sum_{j=1}^{q}\left[f\left(n_{0}+m_{j}\right)-f\left(n_{0}\right)\right] \\
= & -\sum_{i=1}^{p} \sum_{k=1}^{l_{i}} f^{\prime}\left(n_{0}-k\right) \\
& +\sum_{j=1}^{q} \sum_{k=1}^{m_{j}} f^{\prime}\left(n_{0}+k-1\right) \\
\leqslant & 0 \quad(\text { by Lemma 1). }
\end{aligned}
$$

Therefore, we have

$s f\left(n_{0}\right) \geqslant \sum_{i=1}^{s} f\left(n_{0}+d_{i}\right)$

Lemma 3. Let $f: N^{+} \rightarrow R$ defined by

$f(n)= \begin{cases}c_{1}+c_{2} \sqrt{n} & \text { if } n \leqslant D_{0}, \\ c_{3}+c_{4} n & \text { if } n>D_{0},\end{cases}$

be a function used to model hard disk seek time, where $n$ is the distance of the seek in numbers of cylinders, and $D_{0}$ is a positive integer. Then, we have

$f^{\prime}(i) \geqslant f^{\prime}(j)$ for all $i<j$,

where $f^{\prime}(n)=f(n+1)-f(n)$.

Proof. Let $g: R \rightarrow R$ be a function defined by

$g(x)= \begin{cases}c_{1}+c_{2} \sqrt{x} & \text { if } 0<x \leqslant D_{1}, \\ c_{3}+c_{4} x & \text { if } x>D_{1}, \\ \text { undefined } & \text { if } x \leqslant 0,\end{cases}$

where $D_{1}$ is the distance the disk head has traversed when it is accelerated to its maximum speed. Let $g^{\prime}(x)=d g(x) / d x$ be the differential function of $g(x)$. Since $1 / g^{\prime}(x)$ is the velocity of the disk head when it has traversed distance $x$, we have

$g^{\prime}\left(D_{1}^{-}\right)=\lim _{x \rightarrow D_{1}^{-}} g^{\prime}(x)=\lim _{x \rightarrow D_{1}^{+}} g^{\prime}(x)=g^{\prime}\left(D_{1}^{+}\right)$ and

$$
g^{\prime}(x)= \begin{cases}\frac{1}{2} c_{2} \frac{1}{\sqrt{x}} & \text { if } 0<x<D_{1}, \\ c_{4} & \text { if } x>D_{1}, \\ \frac{1}{2} c_{2} \frac{1}{\sqrt{D_{1}}}=c_{4} & \text { if } x=D_{1} .\end{cases}
$$

It is quite simple to show that

$g^{\prime}(y) \geqslant g^{\prime}(z)$ if $y<z$.

Accordingly, for all $i, j \in N^{+}, i<j$, we have

$$
\begin{aligned}
f^{\prime}(i) & =f(i+1)-f(i)=g(i+1)-g(i) \\
& =\int_{i}^{i+1} g^{\prime}(x) d x \geqslant \int_{j}^{j+1} g^{\prime}(x) d x \\
& =g(j+1)-g(j)=f(j+1)-f(j) \\
& =f^{\prime}(j) .
\end{aligned}
$$

Theorem 4. If a disk head scans across a region of $C$ cylinders and make $(s-1)$ stops, then the lumped seek time is maximized when the $(s-1)$ stops are evenly apart by $C / s$. Here, it is assumed that $C$ is a multiple of $s$.

Proof. Let $n_{0}=C / s$ and $d_{1}, d_{2}, \ldots, d_{s}$ be a series of integer with $\sum_{i=1}^{s} d_{i}=0$ and $n_{0}+d_{i} \geqslant 1$ for all $1 \leqslant$ $i \leqslant s$. Let $f: N^{+} \rightarrow R$ be a function of the general form shown in Lemma 3 that models the disk seek time. Then, by Lemmas 2 and 3, we have

$s f\left(n_{y 0}\right) \geqslant \sum_{i=1}^{s} f\left(n_{0}+d_{i}\right)$.

This means the lumped seek time is maximized when the $(s-1)$ stops are evenly apart by $C / s$.

In Theorem 1 above, it is assumed that $C$ is a multiple of $s$. If it is not the case, then the designer can use $[C / s\rceil$ instead.

\section{Conclusion}

In this paper, a tight upper bound of the lumped disk seek time for the Scan policy based on an accurate disk seek time model is presented. The tight upper 
bound states that, when a disk head scans across a region on the disk surface, the lumped seek time is maximized when the stops are evenly apart. One of the most important applications of the tight upper bound is to improve disk bandwidth utilization in multimedia storage system design.

\section{References}

11] E.G. Coffman, L.A. Klimko and B. Ryan, Analysis of scanning policies for reducing disk seek times, SIAM J. Comput. 1 (3) (1972).

12] R. Geist and S. Daniels, A continuum of disk scheduling algorithms, ACM Trans. Comput. Systems 5 (1) (1987).
[3] A.L. Narasimha Reddy and J.C. Wyllie, I/O issues in a multimedia system, IEEE Comput. 27 (3) (1994) 69-74.

[4] C. Ruemmler and J. Wilkes, An introduction to disk drive modeling, IEEE Comput. 27 (3) (1994) 17-28.

[5] T.J. Teorey and T.B. Pinkerton, A comparative analysis of disk scheduling policies, Comm. ACM 15 (3) (1972).

[6] H.M. Vin and P.V. Rangan, Designing file systems for digital video and audio, in: Proc. 13th ACM Symp. on Operating Sysiem Frinciples (1991) 81-94.

[7] P.S. Yu, M.S. Chen and D.D. Kandlur, Design and analysis of a grounded sweeping scheme for multimedia storage management, un: Proc. 3rd Internat. Workshop on Network and Operating System Support for Audio and Video, 1992. 